\title{
ON THE SOLUTIONS OF THE MATHIEU'S EQUATION
}

\author{
Dao Huy Bich ${ }^{1}$, Nguyen Dang Bich ${ }^{2}$, Nguyen Anh Tuan ${ }^{2}$ \\ ${ }^{1}$ Vietnam National University, Hanoi, Vietnam \\ ${ }^{2}$ Institute for Building Science and Technology, Hanoi, Vietnam
}

\begin{abstract}
As shown in [1] solutions of the Mathieu's equation were classified on three fundamental kinds depending mainly on its parameters. These solutions were constructed in the form of infinite series. This paper presents a new approach in which approximated analytical solutions of the Mathieu's equation are constructed in the finite form. Depending on parameters of Mathieu's equations general solutions may obtain following behaviors: either bounded almost periodic, or infinitely increased combining with infinitely decreased and or infinitely increased combining with periodic.
\end{abstract}

\section{INTRODUCTION}

Consider a Mathieu's equation

$$
\ddot{x}+\omega^{2}(k+p \cos \omega t) x=0
$$

solutions of which have important role in investigation of stability problems and in searching solutions of other non-linear differential equations. Consequently, since 1947 McLachlan N.W.[1] presented methods for constructing solutions of the equation (1) and showed the stability domain of solutions in plane $(k, p)$ [see 1, pp. 40-41]. These results later were referenced in works of Kauderer 1961 [see 2, pp. 572-573] and Nguyen Van Dao et al. 2005 [see 3, p.123]. Solution kinds of the Mathieu's equation and their stability domain are fundamental scientific results taking attention of many scientists, but to now there is a little similar research.

The stability domain of solution in plane $(k, p)$ was defined by curves of the form

$$
k=m^{2}+\alpha_{1} p+\alpha_{2} p^{2}+\alpha_{3} p^{3}+\ldots, \quad m=0,1,2,3, \ldots
$$

where $\alpha_{1}, \alpha_{2}, \alpha_{3} \ldots$ are coefficients to be determined in the solving process. The relations (2) are so-called characteristic relations. For example, the characteristic relation corresponds $m=1[1]$

$$
k=1+p-\frac{1}{8} p^{2}-\frac{1}{64} p^{3}-\frac{1}{1536} p^{4}+\frac{11}{36864} p^{5}+0\left(p^{6}\right),
$$


according to which the expression of solution is

$$
\begin{aligned}
x= & \cos t-\frac{1}{8} p \cos 3 t+\frac{1}{64} p^{2}\left(-3 \cos 3 t+\frac{1}{3} \cos 5 t\right) \\
& -\frac{1}{512} p^{3}\left(\frac{1}{3} \cos 3 t-\frac{4}{9} \cos 5 t+\frac{1}{18} \cos 7 t\right) \\
& +\frac{1}{4096} p^{4}\left(\frac{11}{9} \cos 3 t+\frac{1}{6} \cos 5 t-\frac{1}{12} \cos 7 t+\frac{1}{180} \cos 9 t\right)+0\left(p^{5}\right) .
\end{aligned}
$$

Note that expressions (3), (4) and other similar expressions corresponding to different values $\mathbf{m}$ were presented in infinite series.

In the present paper the authors propose a new approach in formulation of characteristic relations and approximated analytical solutions of the Mathieu's equation in the finite form.

\section{IDEA IN FORMULATION OF AN APPROXIMATED ANALYTICAL SOLUTION}

Consider an equation

$$
\ddot{u}+\omega^{2} h(t) u=0 .
$$

In general it has no an exact solution, except the case when $h(t)$ has special form such as

$$
h(t) \equiv h_{1}(t)=\frac{2\left(\frac{\lambda^{2}}{\beta^{2}}-1\right)}{\left(\frac{\lambda}{\beta}+\cos \omega t\right)^{2}}-\frac{2\left(\frac{\lambda^{2}}{\beta^{2}}-1\right) \frac{\alpha \beta}{\omega^{2}}+3 \frac{\lambda}{\beta}}{\frac{\lambda}{\beta}+\cos \omega t}+\frac{2\left(\frac{\lambda^{2}}{\beta^{2}}-1\right) \frac{\alpha \beta}{\omega^{2}}+3 \frac{\lambda}{\beta}+\frac{\omega^{2}}{\alpha \beta}}{\frac{\omega^{2}}{\alpha \beta}+\frac{\lambda}{\beta}+\cos \omega t}
$$

and Eq.(5) has an exact solution [5]

$$
u=\frac{\frac{\omega^{2}}{\alpha \beta}+\frac{\lambda}{\beta}+\cos \omega t}{\frac{\lambda}{\beta}+\cos \omega t}\left[C_{1}+C_{2} \int_{0}^{t} \frac{\left(\frac{\lambda}{\beta}+\cos \omega t\right)^{2} d t}{\left(\frac{\omega^{2}}{\alpha \beta}+\frac{\lambda}{\beta}+\cos \omega t\right)^{2}}\right] .
$$

When $h(t) \equiv g_{1}(t)=k_{1}+p_{1} \cos \omega t$, the equation (5) may have not an exact solution, but it may be seen that (7) is an approximated analytical solution when functions $h_{1}(\mathrm{t})$ and $g_{1}(t)$ are equal approximately to each other with all $\mathbf{t}$. For the reason $h_{1}(t)$ and $g_{1}(t)$ are equal approximately to each other with all $\mathbf{t}$, it is necessary to satisfy following conditions: (i) Values of functions $\mathrm{h}_{1}(\mathrm{t})$ and $\mathrm{g}_{1}(\mathrm{t})$ at extremum points $t=\frac{2 n \pi}{\omega}$ and $t=\frac{(2 n+1) \pi}{\omega}$ must be equal to each other

$$
h_{1}\left(\frac{2 n \pi}{\omega}\right)=g_{1}\left(\frac{2 n \pi}{\omega}\right), \quad h_{1}\left(\frac{(2 n+1) \pi}{\omega}\right)=g_{1}\left(\frac{(2 n+1) \pi}{\omega}\right) .
$$

(ii) Functions $h_{1}(t)$ and $g_{1}(t)$ have no other extremum points, except points $t=\frac{2 n \pi}{\omega}$ and $t=\frac{(2 n+1) \pi}{\omega}$, i.e.

$$
\frac{d}{d t}\left[h_{1}(t)\right]=f(t) \sin \omega t, \quad \text { with } f(t) \neq 0 \text { for al } t ; \quad \frac{d}{d t}\left[g_{1}(t)\right]=-\omega p_{1} \sin \omega t .
$$


From (8) it follows [5]

$$
\frac{\lambda}{\beta}=\frac{p_{1} \pm \sqrt{\Delta}}{k_{1}^{2}-p_{1}^{2}-k_{1}}, \quad \Delta=\left(k_{1}^{2}-p_{1}^{2}\right)\left(k_{1}^{2}-p_{1}^{2}-1\right),
$$

with sign "+" when $p_{1}>0$ and sign " - " when $p_{1}<0$;

$$
\frac{\omega^{2}}{\alpha \beta}+\frac{\lambda}{\beta}=-\frac{k_{1}^{2}-p_{1}^{2}-k_{1}}{k_{1}^{2}-p_{1}^{2}+k_{1}} \cdot \frac{\lambda}{\beta} ; \quad \frac{\omega^{2}}{\alpha \beta}=-\frac{2\left(k_{1}^{2}-p_{1}^{2}\right)}{k_{1}^{2}-p_{1}^{2}+k_{1}} \cdot \frac{\lambda}{\beta} .
$$

According to condition $\left|\frac{\lambda}{\beta}\right|>1,\left|\frac{\omega^{2}}{\alpha \beta}+\frac{\lambda}{\beta}\right|>1$ and $f(t) \neq 0$, we have [5]

$$
\begin{aligned}
& k_{1}^{2}-p_{1}^{2}<0, \quad\left(k_{1}^{2}-p_{1}^{2}\right)\left(k_{1}^{2}-p_{1}^{2}-1\right)-16 k_{1}^{2} \geq 0, \\
& \left|\left(\frac{4}{3}-\frac{k_{1}^{2}-p_{1}^{2}-k_{1}}{k_{1}^{2}-p_{1}^{2}+k_{1}}\right) \frac{\lambda^{2}}{\beta^{2}}-\frac{1}{3}\right| \geq\left|2 \frac{\lambda}{\beta}\right| .
\end{aligned}
$$

Relations (12) define a domain in plane $\left(\mathrm{k}_{1}, \mathrm{p}_{1}\right)$, which is called a characteristic domain (see Fig. 1).

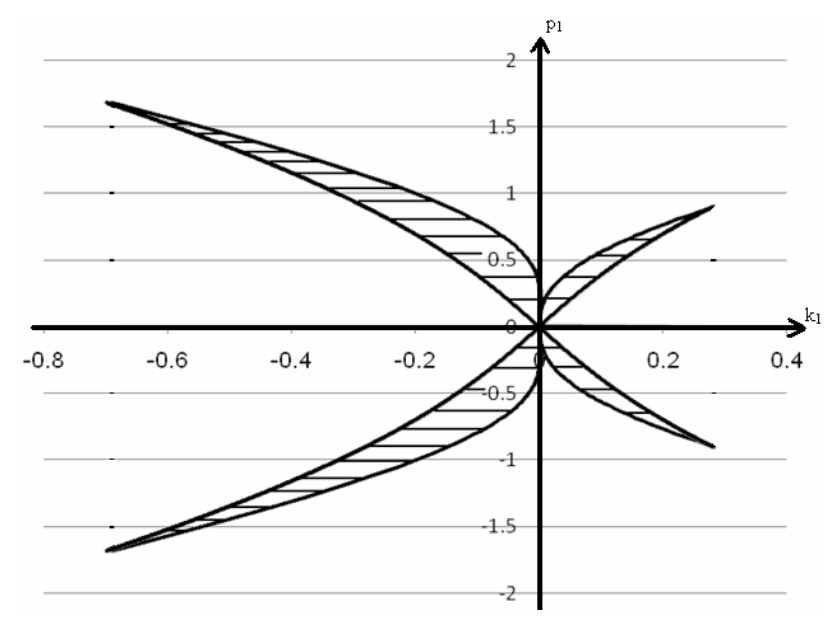

Fig. 1. Characteristic domain

The boundary of characteristic domain is determined by characteristic curves

$$
\begin{aligned}
& \left(k_{1}^{2}-p_{1}^{2}\right)\left(k_{1}^{2}-p_{1}^{2}-1\right)-16 k_{1}^{2}=0, \\
& \left|\left(\frac{4}{3}-\frac{k_{1}^{2}-p_{1}^{2}-k_{1}}{k_{1}^{2}-p_{1}^{2}+k_{1}}\right) \frac{\lambda^{2}}{\beta^{2}}-\frac{1}{3}\right|=\left|2 \frac{\lambda}{\beta}\right| .
\end{aligned}
$$

If $\left(k_{1}, p_{1}\right)$ belongs to the characteristic domain, then the relations $(12)$ are satisfied and Eq. (5) with $h(t)=g_{1}(t)$ :

$$
\ddot{u}+\omega^{2}\left(k_{1}+p_{1} \cos \omega t\right) u=0
$$

has an approximated analytical solution of the form (7).

So that the relations (12) and solution (7) are called new characteristic relations and approximated analytical solution. They are presented in finite form. 


\section{THE KINDS OF SOLUTIONS}

\subsection{Solutions of the first kind}

Conditions for Eq. (1) obtaining a solution of the first kind are $k=k_{1}, p=p_{1}$, where $\left(k_{1}, p_{1}\right)$ belongs to the characteristic domain. Obviously this is the solution in form (7) with $\frac{\lambda}{\beta}$ defined by (10), and $\frac{\omega^{2}}{\alpha \beta}+\frac{\lambda}{\beta}$ - by (11) and the integral term may be evaluated as

$$
\begin{aligned}
& \int_{0}^{t} \frac{\left(\frac{\lambda}{\beta}+\cos \omega t\right)^{2} d t}{\left(\frac{\omega^{2}}{\alpha \beta}+\frac{\lambda}{\beta}+\cos \omega t\right)^{2}}=t-\frac{\left(\frac{\omega^{2}}{\alpha \beta}\right)^{2}}{\omega\left[\left(\frac{\omega^{2}}{\alpha \beta}+\frac{\lambda}{\beta}\right)^{2}-1\right]} \frac{\sin \omega t}{\left(\frac{\omega^{2}}{\alpha \beta}+\frac{\lambda}{\beta}+\cos \omega t\right)} \\
& +\left\{\frac{2\left(\frac{\omega^{2}}{\alpha \beta}+\frac{\lambda}{\beta}\right)\left(\frac{\omega^{2}}{\alpha \beta}\right)^{2}}{\omega\left[\left(\frac{\omega^{2}}{\alpha \beta}+\frac{\lambda}{\beta}\right)^{2}-1\right]^{3 / 2}}-\frac{4 \frac{\omega^{2}}{\alpha \beta}}{\omega\left[\left(\frac{\omega^{2}}{\alpha \beta}+\frac{\lambda}{\beta}\right)^{2}-1\right]^{1 / 2}}\right\} \operatorname{arctg}\left(\operatorname{btg} \frac{\omega}{2} t\right),
\end{aligned}
$$

where $b=\left(\frac{\frac{\omega^{2}}{\alpha \beta}+\frac{\lambda}{\beta}-1}{\frac{\omega^{2}}{\alpha \beta}+\frac{\lambda}{\beta}+1}\right)^{1 / 2}$

Consequently the solution (7) can be rewritten in the form

$$
u=u_{1}(t)\left[C_{1}+C_{2} u_{2}(t)\right]+C_{2} u_{1}(t)\left[t+u_{3}(t)\right]
$$

where

$$
\begin{aligned}
& u_{1}(t)=\frac{\frac{\omega^{2}}{\alpha \beta}+\frac{\lambda}{\beta}+\cos \omega t}{\frac{\lambda}{\beta}+\cos \omega t}, \quad u_{2}(t)=\frac{-\left(\frac{\omega^{2}}{\alpha \beta}\right)^{2}}{\omega\left[\left(\frac{\omega^{2}}{\alpha \beta}+\frac{\lambda}{\beta}\right)^{2}-1\right]} \frac{\sin \omega t}{\left(\frac{\omega^{2}}{\alpha \beta}+\frac{\lambda}{\beta}+\cos \omega t\right)}, \\
& u_{3}(t)=\left\{\frac{2\left(\frac{\omega^{2}}{\alpha \beta}+\frac{\lambda}{\beta}\right)^{2}\left(\frac{\omega^{2}}{\alpha \beta}\right)^{2}}{\omega\left[\left(\frac{\omega^{2}}{\alpha \beta}+\frac{\lambda}{\beta}\right)^{2}-1\right]^{3 / 2}}-\frac{4 \frac{\omega^{2}}{\alpha \beta}}{\omega\left[\left(\frac{\omega^{2}}{\alpha \beta}+\frac{\lambda}{\beta}\right)^{2}-1\right]^{1 / 2}}\right\} \operatorname{arctg}\left(\operatorname{btg} \frac{\omega}{2} t\right) .
\end{aligned}
$$

As seen in (15) the solution in this case consists of two terms: one term increasing infinitely with respect to $\mathbf{t}$ and other periodic term of period $\frac{2 \pi}{\omega}$. The solution (15) is called a solution of the first kind. The integral constants are determined by initial conditions. 
Suppose at $t=0, u(0)=u_{0}, \dot{u}(0)=\dot{u}_{0}$ we obtain

$$
C_{1}=\frac{\frac{\lambda}{\beta}+1}{\frac{\omega^{2}}{\alpha \beta}+\frac{\lambda}{\beta}+1} u_{0}, \quad C_{2}=\frac{\frac{\omega^{2}}{\alpha \beta}+\frac{\lambda}{\beta}+1}{\frac{\lambda}{\beta}+1} \dot{u}_{0}
$$

Now we go on to formulate the solution; when $(k, p)$ does not belong to the characteristic domain. First of all it needs an auxiliary determination.

\subsection{Auxiliary determination}

Consider a special equation

$$
\ddot{u}+\left[\frac{d}{d t}\left(\frac{1}{2} \frac{\dot{a}}{a}\right)-\left(\frac{1}{2} \frac{\dot{a}}{a}\right)^{2}\right] u=0 .
$$

According to [5] this equation has an exact solution

$$
u=\frac{1}{a^{1 / 2}}\left[C_{1}+C_{2} \int_{0}^{t} a d t\right],
$$

where $a(t)$ is an arbitrary function.

When $(k, p)$ does not belong to the characteristic domain, we use a transformation

$$
x=u e^{i \varphi},
$$

where $\mathbf{u}$ is a solution of Eq. (17), $\varphi$ is a function to be determined in the solving process an $\mathbf{i}$ is a properly imaginary number $\left(i^{2}=-1\right)$. we obtain

Substituting (18) into Eq. (1) and equating the real part and imaginary part to null

$$
\begin{aligned}
& \ddot{u}+\omega^{2}(k+p \cos \omega t) u-\dot{\varphi}^{2} u=0, \\
& \ddot{\varphi} u+2 \dot{\varphi} \dot{u}=0 .
\end{aligned}
$$

From Eq. (20) it follows $\dot{\varphi}=\frac{c}{u^{2}}$, where $\mathbf{c}$ is an integral constant. According to (17) we take a particular solution

$$
u=a^{-\frac{1}{2}} \text { and then } \dot{\varphi}=c a .
$$

Eliminating $\ddot{u}$ from Eqs. (17), (19) and taking into account (21) we obtain

$$
\omega^{2}(k+p \cos \omega t)-\left[\frac{d}{d t}\left(\frac{1}{2} \frac{\dot{a}}{a}\right)-\left(\frac{1}{2} \frac{\dot{a}}{a}\right)^{2}\right]=c^{2} a^{2} .
$$

Eq. (22) allows to seek function $a(t)$, but it is difficult to find $a(t)$ satisfying exactly this equation. That is why to find function $a(t)$ satisfying Eq. (22) approximately. For this 
reason we take

$$
a(t)=\frac{\left(\frac{\lambda}{\beta}+\cos \omega t\right)^{2}}{\left(\frac{\omega^{2}}{\alpha \beta}+\frac{\lambda}{\beta}+\cos \omega t\right)^{2}}
$$

then

$$
\frac{d}{d t}\left(\frac{1}{2} \frac{\dot{a}}{a}\right)-\left(\frac{1}{2} \frac{\dot{a}}{a}\right)^{2}=\omega^{2} h_{1}(t)
$$

where $h_{1}(\mathrm{t})$ has the form of expression (6).

If $\left(k_{1}, p_{1}\right)$ belongs to the characteristic domain, then $h_{1}(t)$ may be equal approximately to $g_{1}(t)$ with all $\mathbf{t}$

$$
h_{1}(t) \approx \omega^{2}\left(k_{1}+p_{1} \cos \omega t\right)
$$

Taking into account $(23) \div(25)$ Eq. (22) can be rewritten as following

$$
k-k_{1}+\left(p-p_{1}\right) \cos \omega t \approx \frac{c^{2}}{\omega^{2}} \frac{\left(\frac{\lambda}{\beta}+\cos \omega t\right)^{4}}{\left(\frac{\omega^{2}}{\alpha \beta}+\frac{\lambda}{\beta}+\cos \omega t\right)^{4}} .
$$

Denote $h_{2}(t)=\frac{c^{2}}{\omega^{2}} \frac{\left(\frac{\lambda}{\beta}+\cos \omega t\right)^{4}}{\left(\frac{\omega^{2}}{\alpha \beta}+\frac{\lambda}{\beta}+\cos \omega t\right)^{4}}, \quad g_{2}(t)=k-k_{1}+\left(p-p_{1}\right) \cos \omega t$.

Repeating similar discussion as in previous section 2 in order that functions $\mathrm{k}_{2}(\mathrm{t})$ and $g_{2}(t)$ may be equal approximately to each other with all $\mathbf{t}$, the following conditions must be satisfied.

$$
k-k_{1}+p-p_{1}=\frac{c^{2}}{\omega^{2}} \frac{\left(\frac{\lambda}{\beta}+1\right)^{4}}{\left(\frac{\omega^{2}}{\alpha \beta}+\frac{\lambda}{\beta}+1\right)^{4}}, k-k_{1}-\left(p-p_{1}\right)=\frac{c^{2}}{\omega^{2}} \frac{\left(\frac{\lambda}{\beta}-1\right)^{4}}{\left(\frac{\omega^{2}}{\alpha \beta}+\frac{\lambda}{\beta}-1\right)^{4}}
$$

Relations (27) can be rewritten in the other form

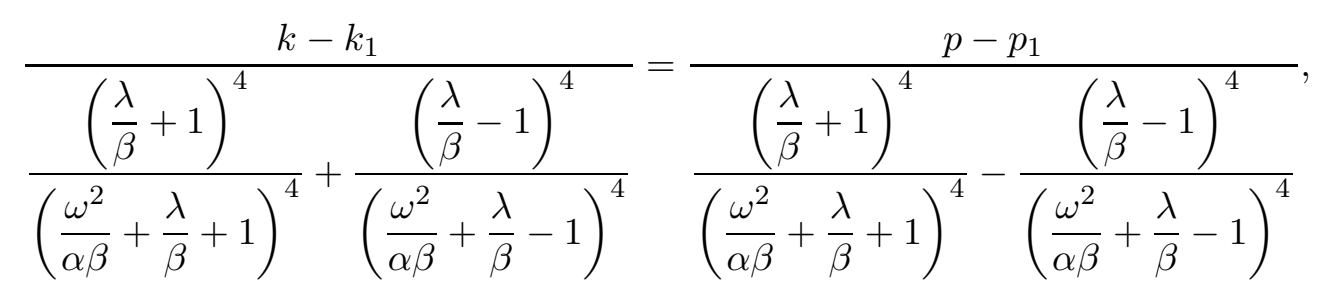




$$
c=\frac{\omega \sqrt{2}\left(k-k_{1}\right)^{1 / 2}}{\left[\frac{\left(\frac{\lambda}{\beta}+1\right)^{4}}{\left(\frac{\omega^{2}}{\alpha \beta}+\frac{\lambda}{\beta}+1\right)^{4}}+\frac{\left(\frac{\lambda}{\beta}-1\right)^{4}}{\left(\frac{\omega^{2}}{\alpha \beta}+\frac{\lambda}{\beta}-1\right)^{4}}\right]^{1 / 2}},
$$

where $\frac{\lambda}{\beta}$ and $\frac{\omega^{2}}{\alpha \beta}+\frac{\lambda}{\beta}$ in own turn depend on $k_{1}, p_{1}$ such as expression (10) and (11). Relations (28) and (29) are called characteristic relations differing from ones in [1], here they have the finite form.

When a couple of parameters $(k, p)$ and Eqs. (10), (11) are given, then the equation (28) is an equation of two variables $k_{1}, p_{1}$, where $k_{1}$ changes in the range [-0.7;0], $p_{1}-$ in the range $[-1.684 ; 1.684]$ and $k_{1}$ - in the range $[0 ; 0.28098], p_{1}-$ in the range $[-0.8998$; 0.8998 ] (see Fig. 1). It is necessary to find $\left(k_{1}, p_{1}\right)$ belonging to the characteristic domain and satisfying Eq. (28). In the principle we can draw the curve $k_{1}-p_{1}$ according to Eq. (28). If this curve intersects the characteristic domain, so we can choose a couple $\left(k_{1}, p_{1}\right)$ satisfying the proposed desire. But in reality, it is difficult to draw the curve $k_{1}-p_{1}$ because of complexity in formulation of this curve from complicated relations (10), (11), and (28). Therefore, instead of the choice $\left(k_{1}, p_{1}\right)$ satisfying the proposed desire we consider either this curve intersects the boundary of characteristic domain or not. Consequently, it leads to find a solution of the set of equations (28) and (13) or (28) and (14). A numerical program was formulated for solving this set of equations to give $k_{1}, p_{1}$ and then $\mathbf{c}$ according to Eq. (29).

Table 1. Some results obtained from formulated program

\begin{tabular}{|c|c|c|c|c|}
\hline $\mathrm{k}$ & $\mathrm{p}$ & $\mathrm{k}_{1}$ & $\mathrm{p}_{1}$ & $\left(\frac{c}{\omega}\right)^{2}$ \\
\hline-0.7 & 0.45 & -0.0325958 & 0.133351788 & -0.00594 \\
\hline-0.7 & 0.854 & -0.0838084 & 0.330159378 & $-2.6 .10^{-5}$ \\
\hline 0.5 & 0.386 & -0.551283432 & 1.437018653 & 0.000888 \\
\hline 0.5 & 0.425 & -0.6068069 & 1.531798374 & 0.000774 \\
\hline
\end{tabular}

From the results in the Table 1, we can see that points $(k, p)$ lie outside the characteristic domain, but points $\left(k_{1}, p_{1}\right)$ belong to the characteristic domain, they lie on the boundary curve (13) of the characteristic domain. The value of $\mathbf{c}$ may be taken as a real number or a properly imaginary number.

\subsection{Solutions of other kinds}

When $(k, p)$ does not belong to the characteristic domain, the Mathieu's equation (1) has solutions of the second and the third kinds.

(i) Solution expressions

According to (18) solution of the Mathieu's equation has the form of a complex function $x=u e^{i \varphi}=u \cos \varphi+i u \sin \varphi$, where $\mathbf{u}$ and $\varphi$ are determined by relations (21) 
and $(23)$

$$
u=\frac{\frac{\omega^{2}}{\alpha \beta}+\frac{\lambda}{\beta}+\cos \omega t}{\frac{\lambda}{\beta}+\cos \omega t}, \dot{\varphi}=c \frac{\left(\frac{\lambda}{\beta}+\cos \omega t\right)^{2}}{\left(\frac{\omega^{2}}{\alpha \beta}+\frac{\lambda}{\beta}+\cos \omega t\right)^{2}}, \text { or } \varphi=c \int_{0}^{t} \frac{\left(\frac{\lambda}{\beta}+\cos \omega t\right)^{2} d t}{\left(\frac{\omega^{2}}{\alpha \beta}+\frac{\lambda}{\beta}+\cos \omega t\right)^{2}} .
$$

By use of expressions (16) function $\varphi$ can be represented as

$$
\varphi=c\left[t+u_{3}(t)+u_{2}(t)\right]
$$

then solutions of the Mathieu's equation may be expressed in three cases as follows

a) Case $c=\theta$, where $\theta$ is a real number

$$
x=\frac{\frac{\omega^{2}}{\alpha \beta}+\frac{\lambda}{\beta}+\cos \omega t}{\frac{\lambda}{\beta}+\cos \omega t}(\cos \bar{\varphi}+i \sin \bar{\varphi})
$$

b) Case $c=i \theta$

c) Case $\mathrm{c}=-\mathrm{i} \theta$

$$
x=\frac{\frac{\omega^{2}}{\alpha \beta}+\frac{\lambda}{\beta}+\cos \omega t}{\frac{\lambda}{\beta}+\cos \omega t} e^{-\bar{\varphi}}
$$

where denote $\bar{\varphi}=\theta\left[t+u_{3}(t)+u_{2}(t)\right]$.

$$
x=\frac{\frac{\omega^{2}}{\alpha \beta}+\frac{\lambda}{\beta}+\cos \omega t}{\frac{\lambda}{\beta}+\cos \omega t} e^{\bar{\varphi}}
$$

Equation (1) is a linear differential equation, from expressions (31)-(33) general solutions of the Mathieu's equation (1) can be represented as follows:

$$
\begin{gathered}
x=\frac{\frac{\omega^{2}}{\alpha \beta}+\frac{\lambda}{\beta}+\cos \omega t}{\frac{\lambda}{\beta}+\cos \omega t}\left(C_{1} \cos \bar{\varphi}+C_{2} \sin \bar{\varphi}\right) \\
x=\frac{\frac{\omega^{2}}{\alpha \beta}+\frac{\lambda}{\beta}+\cos \omega t}{\frac{\lambda}{\beta}+\cos \omega t}\left(C_{1} e^{-\bar{\varphi}}+C_{2} e^{\bar{\varphi}}\right)
\end{gathered}
$$

The solution (34) is a solution of the second kind, which obtains bounded and almost periodic characters. The solution (35) is a solution of the third kind which obtains infinitely increased combining with infinitely decreased characters. 
(ii) Determination of integral constants

From (34) and (35) it follows that

$$
\begin{gathered}
\dot{x}=\frac{\omega^{3} \sin \omega t}{\alpha \beta\left(\frac{\lambda}{\beta}+\cos \omega t\right)^{2}}\left(C_{1} \cos \bar{\varphi}+C_{2} \sin \bar{\varphi}\right)+\left(-C_{1} \sin \bar{\varphi}+C_{2} \cos \bar{\varphi}\right) \theta \frac{\frac{\lambda}{\beta}+\cos \omega t}{\frac{\omega^{2}}{\alpha \beta}+\frac{\lambda}{\beta}+\cos \omega t}, \\
\dot{x}=\frac{\omega^{3} \sin \omega t}{\alpha \beta\left(\frac{\lambda}{\beta}+\cos \omega t\right)^{2}}\left(C_{1} e^{-\bar{\varphi}}+C_{2} e^{\bar{\varphi}}\right)+\left(-C_{1} e^{-\bar{\varphi}}+C_{2} e^{\bar{\varphi}}\right) \theta \frac{\frac{\lambda}{\beta}+\cos \omega t}{\frac{\omega^{2}}{\alpha \beta}+\frac{\lambda}{\beta}+\cos \omega t},
\end{gathered}
$$

Suppose at

$$
t=0 ; \quad x(0)=x_{0}, \quad \dot{x}(0)=\dot{x}_{0}
$$

According to relations (34), (36) and initial conditions (38) the integral constants of the second kind solution can be determined as

$$
C_{1}=\frac{\frac{\lambda}{\beta}+1}{\frac{\omega^{2}}{\alpha \beta}+\frac{\lambda}{\beta}+1} x_{0}, \quad C_{2}=\frac{\left(\frac{\omega^{2}}{\alpha \beta}+\frac{\lambda}{\beta}+1\right)}{\theta\left(\frac{\lambda}{\beta}+1\right)} \dot{x}_{0}
$$

In accordance of relations (35), (37) and initial conditions (38) the integral constants of the third kind solution may be evaluated as

$$
C_{1}=\frac{1}{2} \frac{\frac{\lambda}{\beta}+1}{\frac{\omega^{2}}{\alpha \beta}+\frac{\lambda}{\beta}+1} x_{0}-\frac{1}{2} \frac{\frac{\omega^{2}}{\alpha \beta}+\frac{\lambda}{\beta}+1}{\theta\left(\frac{\lambda}{\beta}+1\right)} \dot{x}_{0}, C_{2}=\frac{1}{2} \frac{\frac{\lambda}{\beta}+1}{\frac{\omega^{2}}{\alpha \beta}+\frac{\lambda}{\beta}+1} x_{0}+\frac{1}{2} \frac{\frac{\omega^{2}}{\alpha \beta}+\frac{\lambda}{\beta}+1}{\theta\left(\frac{\lambda}{\beta}+1\right)} \dot{x}_{0}
$$

\section{CONDITIONS FOR FINDING APPROXIMATED ANALYTICAL SOLUTIONS}

We summarize two conditions to be satisfied for finding approximated analytical solutions of the Mathieu's equation

(i) Condition 1. Either relations (12) must be satisfied, or point $\left(k_{1}, p_{1}\right)$ belongs to the characteristic domain (see Fig. 1) and/or $h_{1}(t)$ is equal approximately to $g_{1}(\mathrm{t})$ with all $\mathbf{t}$, where $\mathrm{h}_{1}(\mathrm{t})$ is evaluated by $(6)$ and $\mathrm{g}_{1}(\mathrm{t})=\mathrm{k}_{1}+\mathrm{p}_{1} \cos \omega \mathrm{t}$.

(ii) Condition 2. Either relations (28), (29) must be satisfied, or $\mathrm{h}_{2}(\mathrm{t})$ is equal approximately to $\mathrm{g}_{2}(\mathrm{t})$, where

$$
h_{2}(t)=\left(\frac{c}{\omega}\right)^{2} \frac{\left(\frac{\lambda}{\beta}+\cos \omega t\right)^{4}}{\left(\frac{\omega^{2}}{\alpha \beta}+\frac{\lambda}{\beta}+\cos \omega t\right)^{4}}, \quad g_{2}(t)=k-k_{1}+\left(p-p_{1}\right) \cos \omega t .
$$


Herein parameters must satisfy relations (28), (29), for example, parameters k, p, $\mathrm{k}_{1}, \mathrm{p}_{1}$, c are given in the Table 1.

\section{SOLVING PROCEDURE AND APPLIED EXAMPLES}

\subsection{Solving procedure}

Step 1. Input given parameters $k, p, \omega, x_{0}, \dot{x}_{0}$

Step 2. If $k=k_{1}, p=p_{1}$ belongs to the characteristic domain, solution of the first kind is taken according to the formulus (15)

Step 3. If $(\mathrm{k}, \mathrm{p})$ does not belong to the characteristic domain, then by use of numerical program to find $k_{1}, p_{1}$ and c satisfying relations (28), (29)

Step 4. If $k-k_{1}>0$, solution of the second kind is taken according to formulus (34)

Step 5. If $k-k_{1}<0$, solution of the third kind is taken according to formulus (35)

Step 6. Checking conditions for finding approximated analytical solution: $h_{1}(t)$ and $g_{1}(t)$ or $h_{2}(t)$ and $g_{2}(t)$ are equal approximately to each other with all $\mathbf{t}$.

\subsection{Applied examples}

The following Figs. $2 \div 7$ present obtained solutions of three kinds of the Mathieu's equation corresponding to different combinations of parameters.

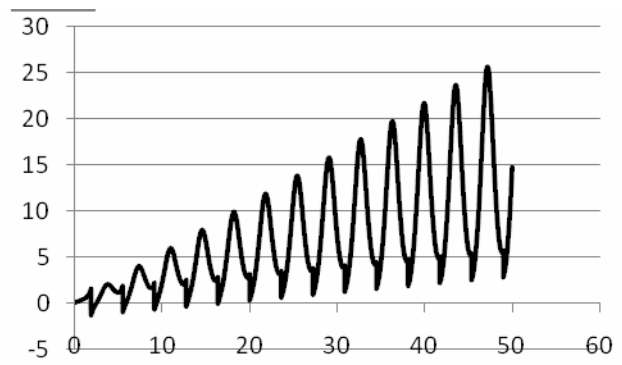

Fig. 2a. Graph of $x(t)$ of $1^{\text {st }}$ kind: $k_{1}=-$ $0.3773536, p_{1}=1.110166042, \omega=1.732$, $x_{0}=0.1, \dot{x}_{0}=0.5$

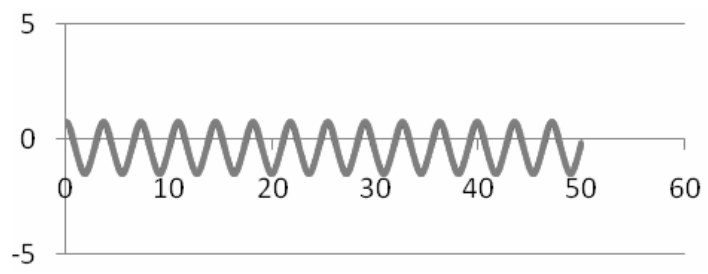

Fig. 2b. Graph of $h_{1}(t)$ and $g_{1}(t)$ of $1^{\text {st }}$ : $k_{1}=-0.3773536, p_{1}=1.110166042, \omega=1.732$, $x_{0}=0.1, \dot{x}_{0}=0.5$

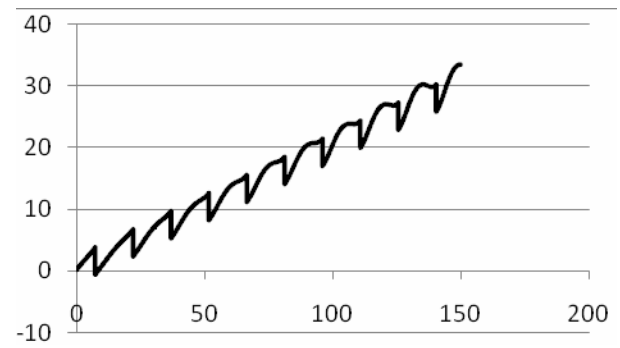

Fig. 3a. Graph of $x(t)$ of $1^{\text {st }}$ kind: $k_{1}=$ 0.01396638, $p_{1}=0.057500748, \omega=0.425$, $x_{0}=0.1, \dot{x}_{0}=0.5$

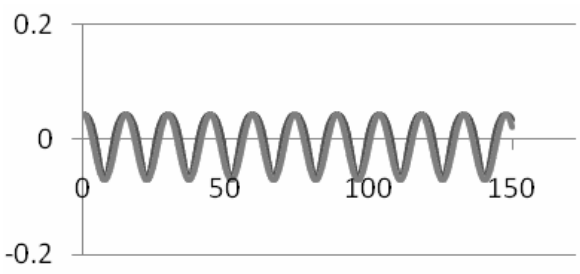

Fig. 3b. Graph of $h_{1}(t)$ and $g_{1}(t)$ of $1^{\text {st }}: k_{1}=-0.01396638, p_{1}=0.057500748$, $\omega=0.425, x_{0}=0.1, \dot{x}_{0}=0.5$ 


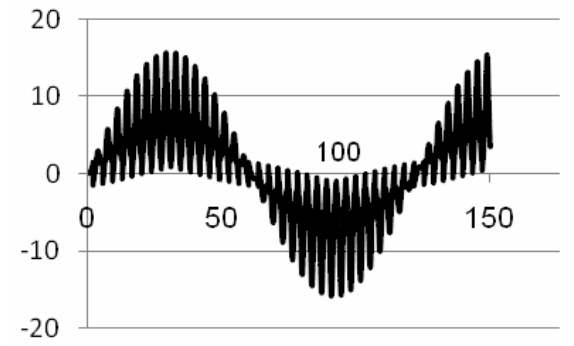

Fig. 4a. Graph $x(t)$ of $2^{\text {nd }}$ kind: $k_{1}=-$ $0.55283432, p_{1}=1.437, k=0.5, p=0.234$, $\omega=1.732, x_{0}=0.1, \dot{x}_{0}=0.5$

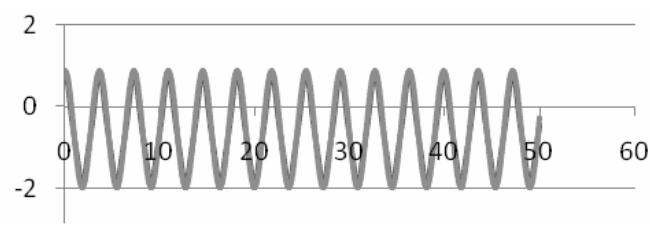

Fig. $4 b$. Graph $h_{1}(t)$ and $g_{1}(t)$ of $2^{\text {nd }}$ kind: $k_{1}=-0.55283432, p_{1}=1.437, k=0.5$, $p=0.234, \omega=1.732, x_{0}=0.1 \dot{x}_{0}=0.5$

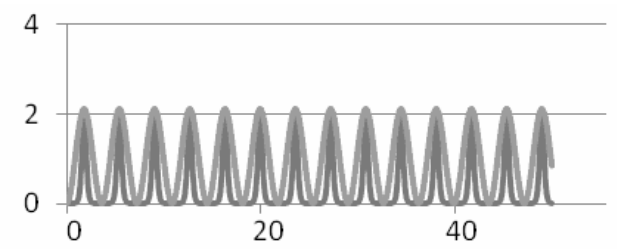

Fig. 4c. Graph $h_{2}(t)$ and $g_{2}(t)$ of $2^{\text {nd }}$ kind: $k_{1}=-0.55283432, p_{1}=1.437, k=0.5$, $p=0.386, \omega=1.732, x_{0}=0.1 \dot{x}_{0}=0.5$



Fig. 6a. Graph $x(t)$ of $3^{\text {rd }}$ kind: $k_{1}=-$ $0.0325958, p_{1}=0.133351788, k=-0.7$, $p=0.45, \omega=0.425, x_{0}=0.1, \dot{x}_{0}=0.5$

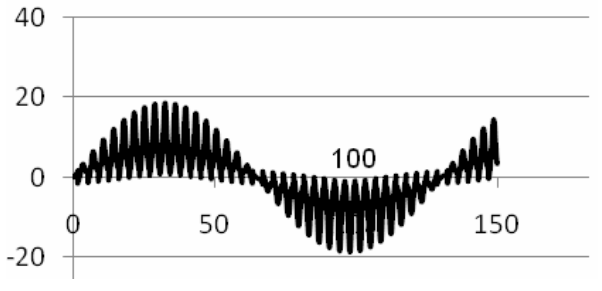

Fig. 5a. Graph $x(t)$ of $2^{\text {nd }}$ kind: $k_{1}=-$ $0.6068069, \quad p_{1}=1.531798374, k=0.5$, $p=0.425, \omega=1.732, x_{0}=0.1, \dot{x}_{0}=0.5$

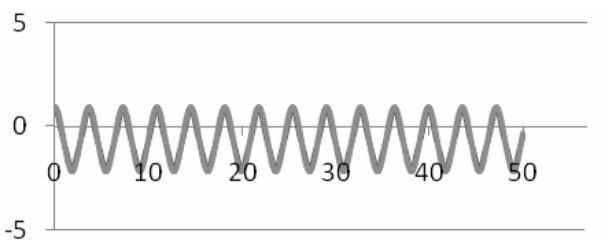

Fig. 5b. Graph $h_{1}(t)$ and $g_{1}(t)$ of $2^{\text {nd }}$ kind: $k_{1}=-0.6068069, p_{1}=1.531798374, k=0.5$, $p=0.425, \omega=1.732, x_{0}=0.1 \dot{x}_{0}=0.5$

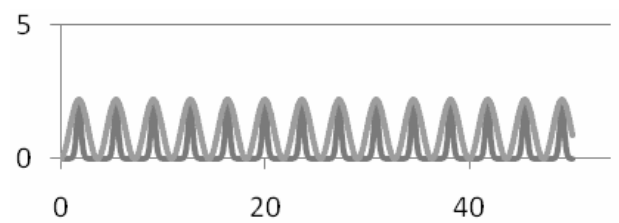

Fig. 5c. Graph $h_{2}(t)$ and $g_{2}(t)$ of $2^{\text {nd }}$ kind: $k_{1}=-0.6068069, p_{1}=1.531798374, k=0.5$, $p=0.425, \omega=1.732, x_{0}=0.1 \dot{x}_{0}=0.5$

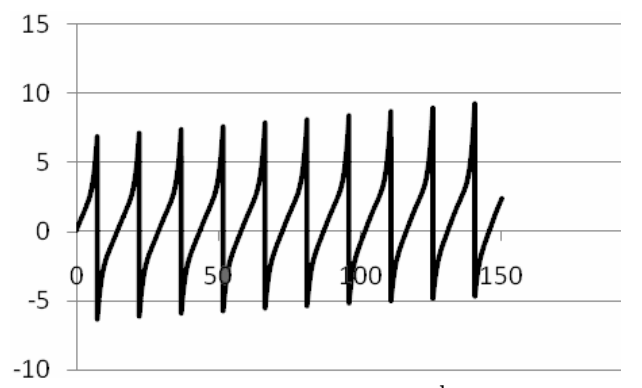

Fig. 7a. Graph $x(t)$ of $3^{\text {rd }}$ kind: $k_{1}=-$ $0.0838084, p_{1}=0.330159378, k=-0.7$, $p=0.854, \omega=0.425, x_{0}=0.1, \dot{x}_{0}=0.5$ 


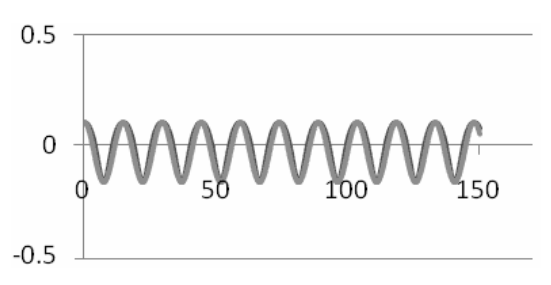

Fig. 6b. Graph $g_{1}(t)$ and $h_{1}(t)$ of $3^{\text {rd }}$ kind: $k_{1}=-0.0325958, p_{1}=0,133351788, k=$ $-0.7, p=0.45, \omega=0.425, x_{0}=0.1 \dot{x}_{0}=0.5$

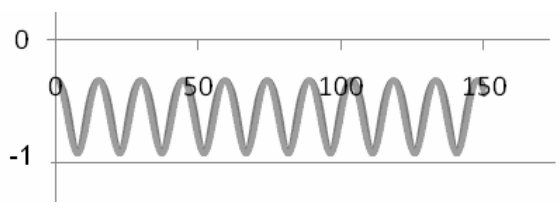

Fig. 6c. Graph $g_{2}(t)$ and $h_{2}(t): k_{1}=-$ $0.0325958, p_{1}=0.133351788, k=-0.7$, $p=0.45, \omega=0.425, x_{0}=0.1 \dot{x}_{0}=0.5$

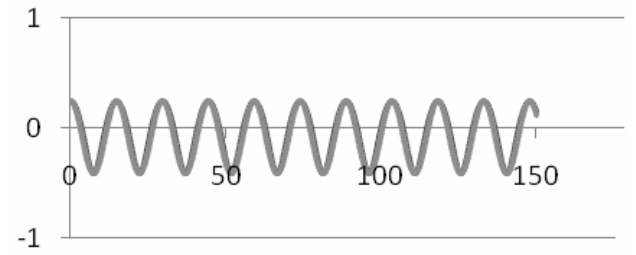

Fig. 7b. Graph $g_{1}(t)$ and $h_{1}(t)$ of $3^{\text {rd }}$ kind: $k_{1}=-$ $0.0838084, p_{1}=0.330159378, k=-0.7, p=0.854$, $\omega=0.425, x_{0}=0.1 \dot{x}_{0}=0.5$

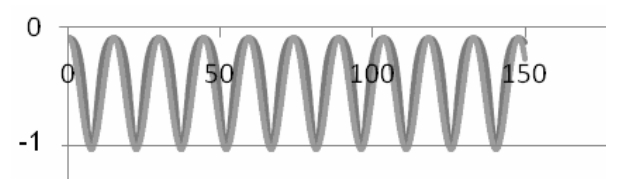

Fig. 7c. Graph $g_{2}(t)$ and $h_{2}(t)$ of $3^{\text {rd }}$ kind: $k_{1}=-$ $0.0838084, p_{1}=0,330159378, k=-0.7, p=0.854$, $\omega=0.425, x_{0}=0.1 \dot{x}_{0}=0.5$

\section{DISCUSSION}

Formulation of the new characteristic relations and approximated analytical solutions in the finite form is derived.

The behaviors of solutions of the first, second, and third kind obtained in this paper are the same behaviors of solutions described in [1] respectively.

Conditions for finding approximated analytical solutions in considered examples are checked and satisfied.

A continuing study must be improved in formulation of the program for solving equations (28) and (29).

Finding a(t) in the form (23) is only an attempt, it may be proposed in other form such that Eq. (22) is better satisfied approximately.

\section{REFERENCES}

[1] N.W. McLachlan, Theory and Application of Mathieu Functions, Oxford at the Clarendon Press, 1947.

[2] G. Kauderer, Non-linear Mechanics, Edition of Foreign Literature, Moscowm (in Russian) 1961.

[3] Nguyen Van Dao, Tran Kim chi, Nguyen Dung. Introduction to Non-linear Dynamics and Chaos, VNU Publishing House (in Vietnamese), 2005.

[4] Dao Huy Bich, Nguyen Dang Bich, On the lateral oscillation problem of beams subjected to axial load, VNU Journal of Science, Math-Phys 20 (4) (2004) 1-10.

[5] Nguyen Dang Bich, Ngo Dinh Bao Nam, Conditions for the approximated analytical solution of a parametric oscillation problem described by the Mathieu equations, VNU Journal of Science, Math.Phys 21 (2) (2006) 9-16. 


\section{VỀ NGHIỆM CỦA PHƯƠNG TRÌNH MATHIEU}

Như đã biết trong công trình của N. W. McLachlan các nghiệm của phương trình Mathieu được chia làm ba dạng cơ bản tùy thuộc vào các tham số của phương trình. Các nghiệm này được thiết lập dưới dạng chuỗi vô hạn. Bài này trình bầy một cách tiếp cận mới xây dung nghiệm giải tích gần đúng dưới dạng hữu hạn của phương trình Mathieu. Tùy thuộc các tham số của phương trình mà nghiệm tổng quát của chúng có thể có tính chất sau: hoặc là giới nội hầu hoàn toàn, hoặc là tăng giảm vô hạn và hoặc là tuần hoàn tăng vô hạn. 